\title{
Nod Factors of Rhizobium leguminosarum bv. viciae and Their Fucosylated Derivatives Stimulate a Nod Factor Cleaving Activity in Pea Roots and Are Hydrolyzed In Vitro by Plant Chitinases at Different Rates
}

\author{
Alexandra O. Ovtsyna, ${ }^{1}$ Michael Schultze, ${ }^{2}$ Igor A. Tikhonovich, ${ }^{1}$ Herman P. Spaink, ${ }^{3}$ \\ Éva Kondorosi, ${ }^{2}$ Ádám Kondorosi, ${ }^{2}$ and Christian Staehelin ${ }^{2}$ \\ ${ }^{1}$ All-Russia Research Institute for Agricultural Microbiology, Podbelsky shosee 3, 189620, St. Petersburg, \\ Pushkin - 8, Russia; ${ }^{2}$ Institut des Sciences Végétales, CNRS, Avenue de la Terrasse, F-91198 \\ Gif-sur-Yvette Cedex, France; ${ }^{3}$ Institute of Molecular Plant Sciences, Clusius Laboratory, \\ Leiden University, Wassenaarseweg 64, 2333 AL Leiden, The Netherlands \\ Accepted 13 April 2000.
}

Nod factors (NFs) are rhizobial lipo-chitooligosaccharide signals that trigger root nodule development in legumes. Modifications of NF structures influence their biological activity and affect their degradation by plant chitinases. Nodulation of certain pea cultivars by Rhizobium leguminosarum bv. viciae requires modification of NFs at the reducing end by either an $\boldsymbol{O}$-acetyl or a fucosyl group. Fucosylated NFs were produced by an in vitro reaction with NodZ fucosyltransferase and purified. Their biological activity on pea was tested by measuring their capacity to stimulate the activity of a hydrolase that cleaves NFs. Nonmodified and fucosylated NFs displayed this activity at nano- to picomolar concentrations, while a sulfated NF from Sinorhizobium meliloti was inactive. In an additional series of experiments, the stability of non-modified and fucosylated NFs in the presence of purified tobacco chitinases was compared. The presence of the fucosyl group affected the degradation rates and the accessibility of specific cleavage sites on the chitooligosaccharide backbone. These results suggest that the fucosyl group in NFs also weakens the interaction of NFs with certain chitinases or chitinase-related proteins in pea roots.

The symbiotic interaction between rhizobia and legumes resulting in the formation of nitrogen-fixing root nodules is a host-specific process. Host specificity is largely determined by the structure of bacterial signal molecules called Nod factors (NFs). All NFs are lipo-chitooligosaccharides, i.e., oli-

Corresponding author: Christian Staehelin; Current address: Laboratoire de Biologie Moléculaire des Plantes Supérieures, Université de Genève, 1 ch. de l'Impératrice, 1292 Chambésy/Genève, Switzerland; Telephone: (+41) (22) 90617 45, Fax: (+41) (22) 9061741

E-mail: staeheli@sc2a.unige.ch

Current address of Michael Schultze: The Plant Laboratory, Department of Biology, University of York, Heslington, York YO10 5YW, U.K. gomers of $\mathrm{N}$-acetylglucosamine, $\mathrm{N}$-linked with a fatty acid at the nonreducing end (Dénarié et al. 1996; Spaink 1996; Schultze and Kondorosi 1998). Nodulation of certain pea cultivars (e.g., Afghan peas) is influenced by structural modifications of the NFs produced by Rhizobium leguminosarum bv. viciae (NodRlv factors) and by a single genetic locus $s y m 2^{A}$ of the host plant genome (Lie 1984). For the establishment of efficient symbiosis between peas harboring $s y m 2^{A}$ and specific $R$. leguminosarum bv. viciae strains, such as strain TOM, production of NodRlv factors carrying an $O$-acetyl group at the reducing end is required (Firmin et al. 1993). Interestingly, fucosylation at the same position of the reducing end of NodRlv factors can functionally replace O-acetylation for nodulation of peas harboring $s y m 2^{A}$ (Ovtsyna et al. 1998). It has been proposed that $s y m 2^{A}$ encodes an NF receptor (Geurts et al. 1997). Modifications at the reducing end, however, could also play a role in preventing the NFs from binding to plant proteins that are not specific NF receptors, e.g., chitinases or chitooligosaccharide receptors.

Plant chitinases have been described as having a function in defense reactions against pathogens. They hydrolyze $\beta-1,4$ linkages of chitin (homopolymer of $\mathrm{N}$-acetylglucosamine), which is a major structural component of higher fungi and arthropods. Chitinases have been classified according to their primary structure (Neuhaus et al. 1996) as belonging to different glycosyl hydrolase families (Henrissat 1991). Chitinase genes have been shown to be expressed in response to pathogen infection, elicitor treatment, or plant hormones (Collinge et al. 1993). Moreover, chitinases often show a developmentally regulated, tissue-specific induction, e.g., during embryogenesis (De Jong et al. 1992) or nodule development (Staehelin et al. 1992; Vasse et al. 1993; Parniske et al. 1994; Goormachtig et al. 1998; Xie et al. 1999), suggesting a function of chitinases in these processes.

More direct evidence for the involvement of chitinases in nodulation was provided by the finding that rhizobial NFs are substrates for plant chitinases (Staehelin et al. 1994a, 1994b; 
Goormachtig et al. 1998; Minic et al. 1998; Schultze et al. 1998). It has been found that specific chitinase isoforms exhibit distinct substrate specificity and cleavage site preference toward NFs. For example, the sulfate group at the reducing end protects tetrameric NFs from Sinorhizobium meliloti (NodSm factors) against hydrolysis by various chitinases (Staehelin et al. 1994b; Schultze et al. 1998). Degradation products, such as lipo-disaccharides and lipo-trisaccharides, exhibited strongly reduced biological activity on host plants, indicating that chitinases are able to inactivate NFs (Heidstra et al. 1994; Staehelin et al. 1994b). Moreover, expression of a chitinase gene from the bacterium Serratia marcescens in $\mathrm{Si}$ norhizobium strains impeded nodulation (Krishnan et al. 1999).

In the $S$. meliloti-Medicago spp. symbiosis, NFs were able to enhance the activity of a novel "lipo-disaccharide forming" NF hydrolase. This enzyme does not exhibit activity toward the polymer chitin. The enzyme activity in the Medicago spp. rhizosphere is specifically stimulated by sulfated NodSm factors at nanomolar concentrations, indicating that active NFs induce their rapid degradation after perception by the host plant (Staehelin et al. 1995, 1997).

In our work presented here, fucosylated derivatives of NodRlv factors were prepared by an in vitro reaction with NodZ fucosyltransferase, then the capacity of these molecules to elicit an increased activity of a NF hydrolase in pea roots was investigated. The enzyme activity was stimulated by nonmodified and fucosylated NodRlv factors to a similar extent, while sulfated NodSm factors were inactive. In a series of in vitro experiments, fucosylated and non-modified NFs showed differences in their resistance against hydrolysis by purified tobacco chitinases.

\section{RESULTS AND DISCUSSION}

\section{In vitro fucosylation of NodRlv factors.}

In the pea-R. leguminosarum bv. viciae symbiosis, cultivars harboring $s y m 2^{A}$ are nodulated only by rhizobial strains producing NFs with a structural modification at the reducing end, namely an $O$-acetyl or fucosyl group (Firmin et al. 1993; Ovtsyna et al. 1998). Purification of NFs carrying an $O$-acetyl group at the reducing end was inefficient due to the low levels of these molecules in rhizobial cultures. The putative acetyltransferase, NodX, could not be purified (A. O. Ovtsyna and H. P. Spaink, unpublished results). To obtain purified NodRlv factors modified at the reducing end, the NodZ protein, a fucosyltransferase from Bradyrhizobium japonicum (Quinto et al. 1997), was used for in vitro fucosylation of NodRlv factors.

Non-fucosylated NodRlv factors were isolated from the culture media of the NF-overproducing strain $R$. leguminosarum bv. viciae RBL 5799. Two major peaks with a strong absorbance at $304 \mathrm{~nm}$ were detected by high-performance liquid chromatography (HPLC) analysis (Fig. 1A). They represent tetra- and pentasaccharides, $\mathrm{O}$-acetylated and $\mathrm{N}$-acylated with a polyunsaturated $\mathrm{C}_{18: 4}$ fatty acid at the nonreducing end, as described previously, i.e., $\operatorname{NodRlv}-\operatorname{IV}\left(\mathrm{C}_{18: 4}, \mathrm{Ac}\right)$ and NodRlv-V(C $\mathrm{C}_{18: 4}$, Ac) (Spaink et al. 1991). Fractions containing these NFs were separately collected and used for the in vitro fucosylation reaction catalyzed by the NodZ protein from B. japonicum. To test the efficiency of the reaction, radioactively labeled GDP- $\beta$-L- $\left[1-{ }^{14} \mathrm{C}\right]$ fucose was used and the incorporation of ${ }^{14} \mathrm{C}$ fucose analyzed on thin-layer chromatography (TLC) plates. Two radioactive spots corresponding to

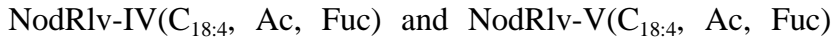
were detected (not shown).

Approximately $100 \mu \mathrm{g}$ of NodRlv factors and an excess of nonradioactive GDP- $\beta$-L-fucose were used in a large-scale reaction for preparative purification by reverse-phase HPLC.

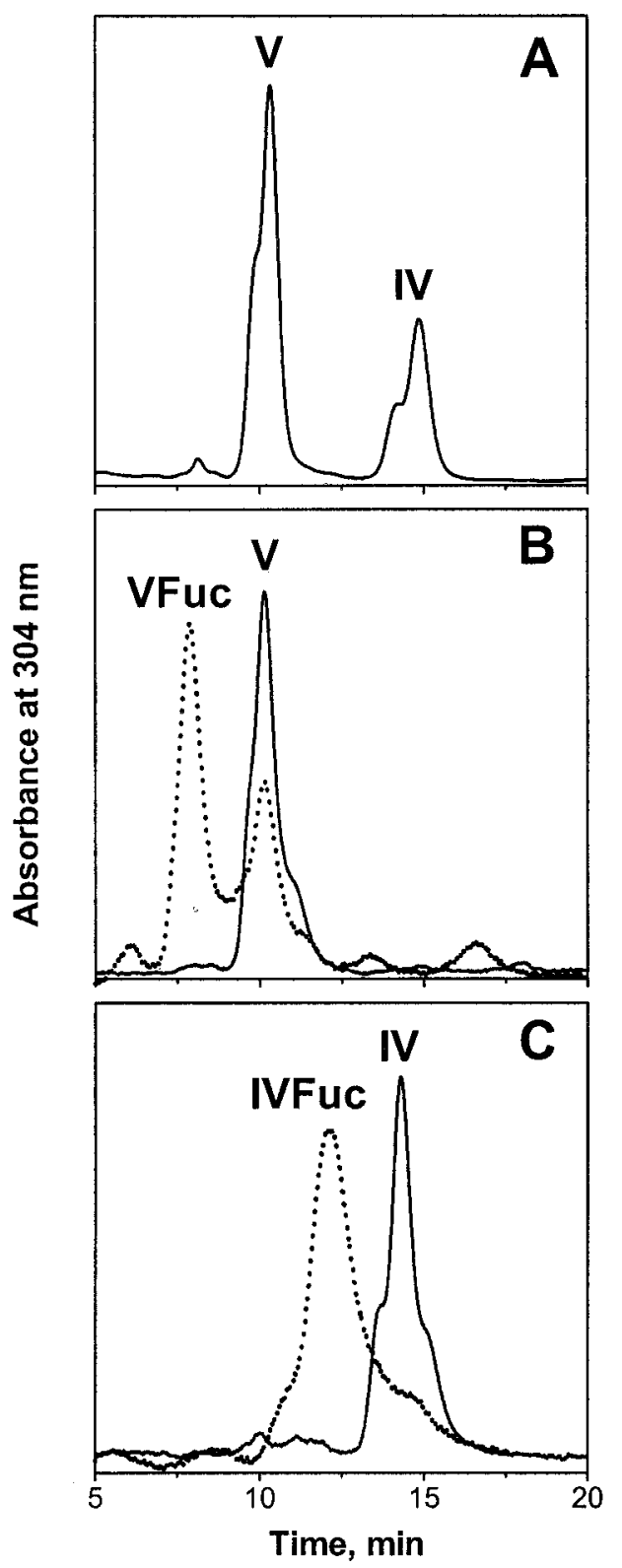

Fig. 1. Purification of Rhizobium leguminosarum bv. viciae (Rlv) Nod factors (NFs) and their fucosylated derivatives. High-performance liquid chromatography (HPLC) analysis was performed with $36 \%$ acetonitrile under isocratic conditions. Absorbance was monitored at $304 \mathrm{~nm}$. A, NFs produced by strain RBL 5799. V: NodRlv-V(C $\left.C_{18: 4}, A c\right)$; IV: NodRlv-IV(C $\left.\mathrm{C}_{18: 4}, \mathrm{Ac}\right)$. B, Solid line: purified NodRlv-V $\left(\mathrm{C}_{18: 4}, \mathrm{Ac}\right)$. Dashed line: NodRlv-V( $\left.\mathrm{C}_{18: 4}, \mathrm{Ac}\right)$ after incubation with the NodZ fucosyltransferase. A new peak with earlier elution time (VFuc) corresponded to the fucosylated derivative NodRlv-V(C $C_{18: 4}$ Ac, Fuc). C, Solid line: purified NodRlv-IV $\left(\mathrm{C}_{18: 4}\right.$, Ac). Dashed line: NodRlv$\operatorname{IV}\left(\mathrm{C}_{18: 4}, \mathrm{Ac}\right)$ after incubation with the NodZ protein. Fucosylation was almost complete. A new peak with earlier elution time (IVFuc) corresponded to the fucosylated derivative NodRlv-IV $\left(\mathrm{C}_{18: 4}, \mathrm{Ac}, \mathrm{Fuc}\right)$. 
As shown in Figure 1B, a new peak corresponding to the fucosylated NodRlv-V( $\mathrm{C}_{18: 4}$, Ac, Fuc $)$ appeared upon incubation with NodZ protein and GDP- $\beta$-L-fucose. The non-fucosylated substrate peak NodRlv-V(C $\mathrm{C}_{18: 4}$, Ac) decreased. Fucosylated tetrameric NodRlv-IV $\left(\mathrm{C}_{18: 4}\right.$, Ac, Fuc $)$ was also formed from the non-fucosylated NodRlv-IV $\left(\mathrm{C}_{18: 4}, \mathrm{Ac}\right)$ substrate (Fig. 1C). Fractions containing the fucosylated NF derivatives were collected and, if required, purified by a second HPLC run under the same conditions.

\section{NodRlv factors and their fucosylated derivatives induce a NF hydrolase activity in pea roots.}

In the $S$. meliloti-Medicago spp. interaction, NFs induce their own degradation by increasing the activity of an NFcleaving plant enzyme. This hydrolytic activity, measured by the release of lipo-disaccharides from NodSm factors, depends on the concentration of NFs that the young seedlings (to be assayed) are preincubated with. The assay has been used to determine the biological activity of a variety of substituted NodSm factors on Medicago sativa (Staehelin et al. 1995). In this work, we examined a similar NF-cleaving hydrolase in pea roots. The biological activity of NodRlv factors and their fucosylated derivatives was measured in pea lines harboring different sym2 alleles.

To induce NF hydrolase activity, roots of pea seedlings were pretreated with Jensen medium containing NFs for $20 \mathrm{~h}$. Control plants were incubated in Jensen medium without NFs. After this preincubation, the seedlings were assayed for their capacity to hydrolyze NodSm-V $\left(\mathrm{C}_{16: 2}, \mathrm{~S}\right)$ at a concentration of $5 \mu \mathrm{M}$. This pentameric NF from $S$. meliloti was found to be a convenient substrate for the NF hydrolase assay of pea. The substrate and acylated cleavage products could be easily recovered from the incubation medium and subsequently analyzed by HPLC as described previously (Staehelin et al. 1994b). Pea roots exhibited a constitutive hydrolytic activity releasing from NodSm-V(C $\left.\mathrm{C}_{16: 2}, \mathrm{~S}\right)$ two acylated cleavage products, i.e., the lipo-trisaccharide $\operatorname{NodSm}-\operatorname{III}\left(\mathrm{C}_{16: 2}\right)$ and the more slowly migrating lipo-disaccharide $\operatorname{NodSm}-\mathrm{II}\left(\mathrm{C}_{16: 2}\right)$. When pea plants were pretreated with NodRlv factors, the lipo-disaccharide forming activity strongly increased, while the lipo-trisaccharide forming activity remained at low levels (Fig. 2B). A similar induction was previously reported for a lipo-disaccharide forming hydrolytic activity of $M$. sativa roots, which was specifically stimulated by sulfated NodSm factors (Staehelin et al. 1995). A pretreatment of pea plants with NodSm-V $\left(\mathrm{C}_{16: 2}, \mathrm{~S}\right)$, i.e., the NF substrate for the enzyme assay did not, however, result in a stimulation of the lipodisaccharide forming hydrolase in both tested pea lines harboring different sym 2 alleles (Fig. 2C). Therefore, NodSm$\mathrm{V}\left(\mathrm{C}_{16: 2}, \mathrm{~S}\right)$ could be used on pea plants as an inert substrate for the NF hydrolase assay.

Figure 3 shows the activity of the NF hydrolase of pea seedlings cv. Rondo, which were pretreated with NodRlv factors or their fucosylated derivatives at different concentrations. After pretreatment, plants were assayed for their NF hydrolase activity with NodSm-V $\left(\mathrm{C}_{16: 2}, \mathrm{~S}\right)$ as the substrate. A mock-treated control plant preincubated without NFs released, per hour, about 45 pmol NodSm-II $\left(\mathrm{C}_{16: 2}\right)$ from NodSm$\mathrm{V}\left(\mathrm{C}_{16: 2}, \mathrm{~S}\right)$. This constitutive activity is indicated in Figure 3 as a horizontal line. A pretreatment with tetrameric NodRlv$\operatorname{IV}\left(\mathrm{C}_{18: 4}, \mathrm{Ac}\right)$ at a concentration of $10^{-6} \mathrm{M}$ resulted in a seven-
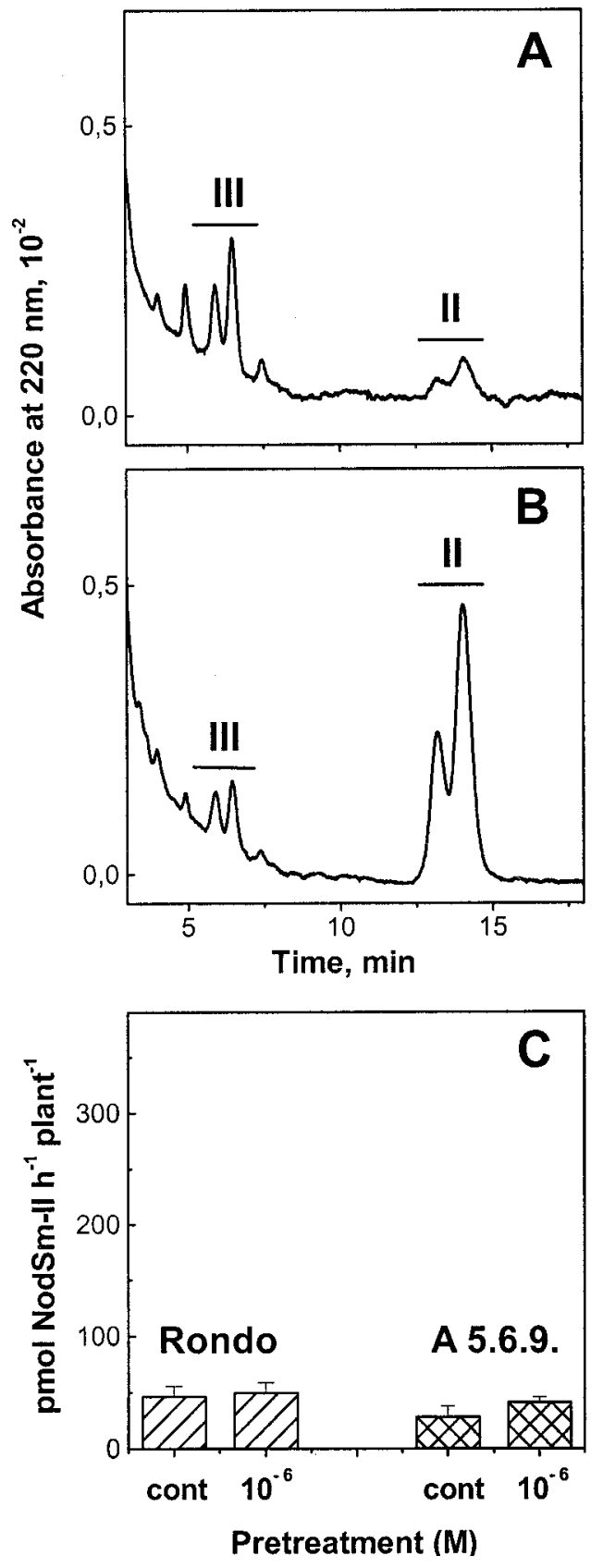

Fig. 2. Hydrolysis of NodSm-V(C $\left.C_{16: 2}, S\right)$ by intact pea roots after pretreatment of seedlings with NodRlv and NodRm factors. Pea seedlings were mock treated or preincubated with Nod factors (NFs) for $20 \mathrm{~h}$ at $18^{\circ} \mathrm{C}$ and than transferred for the assay into a solution containing $5 \mu \mathrm{M}$ NodSm-V $\left(\mathrm{C}_{16: 2}, \mathrm{~S}\right)$. After incubation for $4 \mathrm{~h}$, NFs and their acylated cleavage products were analyzed by high-performance liquid chromatography HPLC). A, HPLC profile of acylated cleavage products of NodSm-V $\left(\mathrm{C}_{16: 2}, \mathrm{~S}\right)$ formed by mock-treated control plants. III: Lipo-trisaccharide NodSm-III $\left(\mathrm{C}_{16: 2}\right)$; II: Lipo-disaccharide NodSm-II $\left(\mathrm{C}_{16: 2}\right)$. Both compounds were separated into their anomers. B, HPLC profile of the NodSm cleavage products of NodSm-V(C $\left.C_{16: 2}, S\right)$ formed by pea seedlings pretreated with NodRlv factors at a concentration of $10^{-6} \mathrm{M}$. The amount of lipo-trisaccharide (III) remained low, while the peak corresponding the lipo-disaccharide (II) increased. C, Activity of the NodSm-II $\left(\mathrm{C}_{16: 2}\right)$-producing pea hydrolase after pretreatment with $10^{-6} \mathrm{M}$ NodSm-V $\left(\mathrm{C}_{16: 2}, \mathrm{~S}\right)$. In both pea lines (cv. Rondo and $s y m 2^{A}$-carrying line A 5.6.9.), the hydrolytic activities of plants pretreated with $10^{-6} \mathrm{M}$ NodSm-V $\left(\mathrm{C}_{16: 2}, \mathrm{~S}\right)$ (columns marked with $10^{-6}$ ) were similar to those of mock-treated control plants (columns marked with cont). Data are means \pm SE for three plants analyzed independently. 
fold increase of NF hydrolase activity. After pretreatment with lower concentrations, a weaker stimulation was observed. At $10^{-11} \mathrm{M}$, the activity reached the value obtained for mocktreated control plants (Fig. 3A). An inducing effect on the pea NF hydrolase was also observed when the fucosylated derivative was tested. Pretreatment of roots with $10^{-11} \mathrm{M}$ NodRlv$\operatorname{IV}\left(\mathrm{C}_{18: 4}, \mathrm{Ac}, \mathrm{Fuc}\right)$ still resulted in a twofold stimulation of the enzyme (Fig. 3B). This NF was also tested at lower concentrations and a threshold of about $10^{-13} \mathrm{M}$ was determined (data not shown). Pentameric NodRlv-V( $\left.\mathrm{C}_{18: 4}, \mathrm{Ac}\right)($ Fig. $3 \mathrm{C})$ and its fucosylated derivative (Fig. 3D) showed a similar inducing activity. They were less active than the tetrameric molecules, however, in stimulating the lipo-disaccharide forming enzyme. Similar stimulatory effects were obtained when non-modified and fucosylated NodRlv factors were tested on the $s y m 2^{A}$ carrying line A 5.6.9. instead of cv. Rondo (data not shown). These results indicate that, independently of the sym 2 allele, stimulation of the NF hydrolase is an early feedback response to NodRlv factors and their fucosylated derivatives.

In an additional experiment, we examined the capacity of $\mathrm{cv}$. Rondo and the $\operatorname{sym} 2^{A}$ harboring line A 5.6.9. to induce the
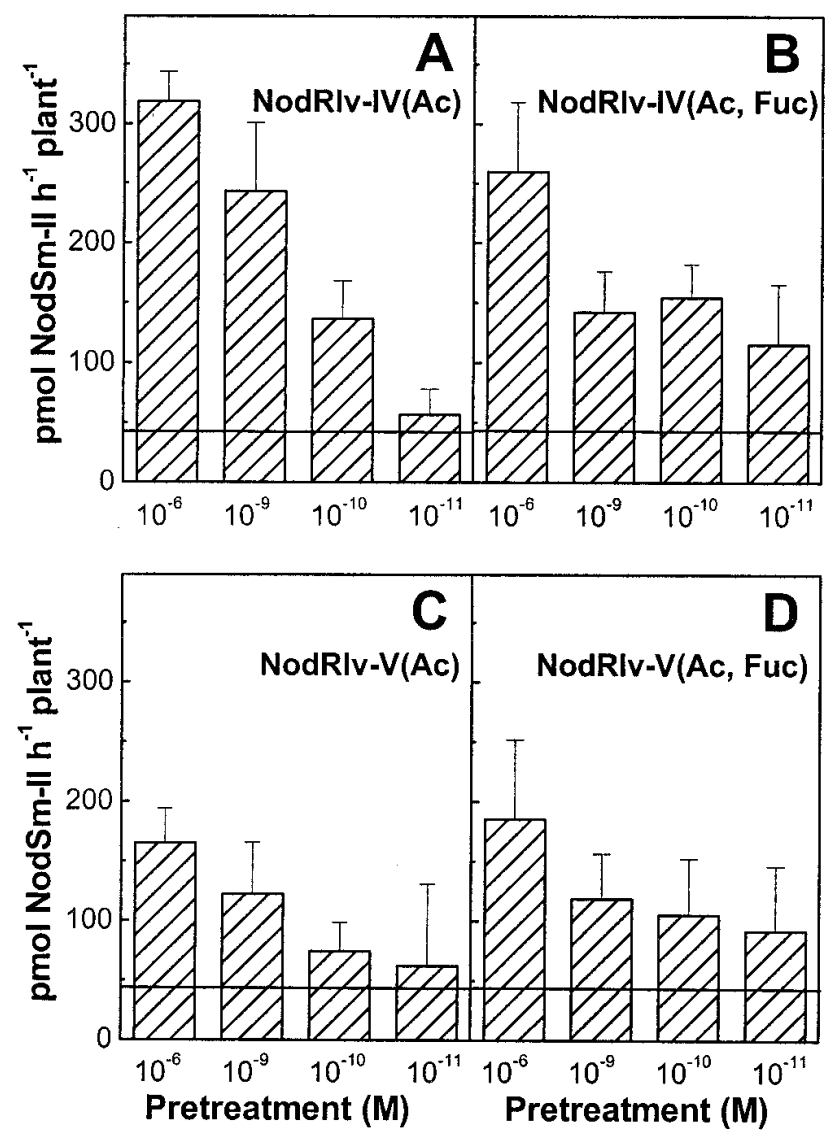

Fig. 3. Activity of the lipo-disaccharide forming pea hydrolase after pretreatment of cv. Rondo with NodRlv factors at different concentrations. Horizontal line indicates activity of lipo-disaccharide formation from NodSm- $\mathrm{V}\left(\mathrm{C}_{16: 2}, \mathrm{~S}\right)$ by a mock-treated control plant (formation of $47 \pm 8$ pmol NodSm-II $\left(C_{16: 2}\right)$ per hour). Data are means \pm SE for three plants analyzed independently. A, Pretreatment with tetrameric NodRlvIV $\left(C_{18: 4}, A c\right)$. B, Pretreatment with fucosylated NodRlv-IV $\left(C_{18: 4}, A c\right.$, Fuc). C, Pretreatment with pentameric NodRlv-V(C $\left.C_{18: 4}, A c\right)$. D, Pretreatment with fucosylated NodRlv-V( $\left.\mathrm{C}_{18: 4}, \mathrm{Ac}, \mathrm{Fuc}\right)$.
NF hydrolase after pretreatment with rhizobial cultures. Two $R$. leguminosarum bv. viciae strains differing in their ability to nodulate $s y m 2^{A}$ harboring peas were tested. Strain CIAM 1026 poorly nodulates line A 5.6.9., unlike the compatible strain TOM, which produces NFs carrying a reducing end modification (Firmin et al. 1993). As shown in Figure 4, pretreatment with strain CIAM 1026 clearly enhanced the lipo-disaccharide forming NF hydrolase activity in both pea lines. Strain TOM, however, showed no stimulatory effects in this experiment. A weak induction was found, though, when other pea cultivars were tested (data not shown). It is known that strain TOM produces very low amounts of NFs compared with the levels produced by strain CIAM 1026 (Ovtsyna et al. 1999). Thus, the amount of NFs secreted by strain TOM might not have been sufficient to induce the NF hydrolase in the experiment shown in Figure 4. These findings are consistent with the breakdown of non-modified NodRlv factors in planta, which were hydrolyzed by pea roots independently of the presence of the $\operatorname{sym}^{A}$ allele (Geurts et al. 1997).

These results show that pea plants respond to NodRlv factors and their fucosylated derivatives with the stimulation of an NF cleaving enzyme activity. This response is independent of the $\operatorname{sym} 2^{A}$ allele. The function of the NF-inducible pea hydrolase and its substrate specificity toward non-modified and fucosylated NodRlv factors remains to be determined. We suggest that this enzyme plays a role in a general breakdown of NFs after their perception by the host plant. A reduction of NF levels might be an important step in NF signaling, to avoid putative inhibitory effects induced by continuous stimulation of active NFs (Staehelin et al. 1995).

\section{Fucosylation increases the resistance of NodRlv factors toward hydrolysis by tobacco chitinases.}

Previous work demonstrated that modifications in NFs affect their stability against chitinase degradation, thereby influencing the actual level of active NFs in the rhizosphere and in in vitro experiments. Generally, leguminous and non-legum-
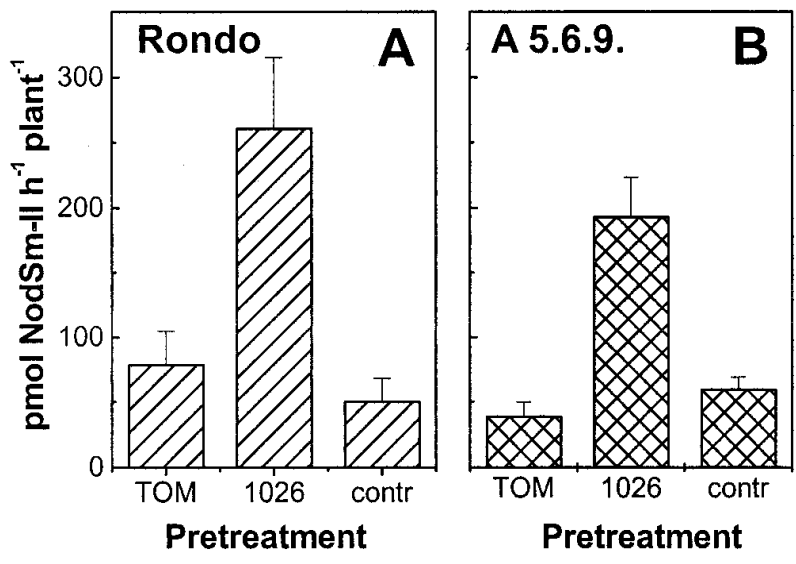

Fig. 4. Stimulation of the lipo-disaccharide forming pea hydrolase after pretreatment with Rhizobium leguminosarum bv. viciae strains. Plants were preincubated in a solution containing strain TOM or strain CIAM 1026 (initial $A_{660}=0.05$ ) for $20 \mathrm{~h}$ at $18^{\circ} \mathrm{C}$ and tested for their lipo-disaccharide formation from NodSm-V $\left(\mathrm{C}_{16: 2}, \mathrm{~S}\right)$. As a control, the hydrolytic activity of seedlings incubated without bacteria was determined (columns marked with contr). Data are means \pm SE for three plants analyzed independently. A, Cv. Rondo. B, sym $2^{A}$-carrying line A 5.6.9. 
inous enzymes belonging to the same chitinase class showed a similar substrate specificity and cleavage site preference toward NodSm factors (Staehelin et al. 1994b; Minic et al. 1998; Schultze et al. 1998). Various class III chitinases (belonging to glycosyl hydrolase family 18) cleaved sulfated and non-sulfated NodSm factors at a similar rate of hydrolysis. The sulfate group at the reducing end, however, strongly protected NFs against hydrolysis by class I chitinases (belonging to glycosyl hydrolase family 19) (Staehelin et al. 1994b; Schultze et al. 1998). To analyze whether the fucosyl group at the reducing end of NodRlv factors affects hydrolysis by plant chitinases in a similar way, we examined a class I chitinase and a class III chitinase for their substrate specificity and cleavage site preference toward purified NodRlv-IV $\left(\mathrm{C}_{18: 4}, \mathrm{Ac}\right)$, NodRlv-V( $\mathrm{C}_{18: 4}$, Ac) and their fucosylated derivatives. After incubation, the substrate and the acylated cleavage products were extracted with $n$-butanol and analyzed by reverse-phase HPLC.

Incubation of pentameric NodRlv-V $\left(\mathrm{C}_{18: 4}, \mathrm{Ac}\right)$ with a class I chitinase (Chi32 from Nicotiana tabacum) gave rise to two acylated products, the lipo-tetrasaccharide $\operatorname{NodRlv-IV(} \mathrm{C}_{18: 4}$, Ac) and the lipo-trisaccharide $\left.\operatorname{NodRlv-III(} \mathrm{C}_{18: 4}, \mathrm{Ac}\right)$ separated into its anomers (Fig. 5A). Purified NodRlv-IV(C $\left.\mathrm{C}_{18: 4}, \mathrm{Ac}\right)$ was not degraded, even after prolonged incubation with the class I chitinase (not shown). The presence of a fucosyl group strongly affected hydrolysis of pentameric substrates. The class I chitinase slowly released the lipo-trisaccharide from NodRlv-V( $\mathrm{C}_{18: 4}$, Ac, Fuc), while the lipo-tetrasaccharide was not formed (Fig. 5B). This result indicates that the fucosyl group makes the neighboring glycosidic bond inaccessible for the class I enzyme. Purified tetrameric NodRlv-IV $\left(\mathrm{C}_{18: 4}, \mathrm{Ac}\right.$, Fuc) was resistant against hydrolysis (not shown).

Figure 5C shows the schematic structure of the tested NFs and their cleavage sites accessible for the class I chitinase. The hydrolytic rate for each glycosidic bond in each NF substrate was determined. This value was expressed as a percentage of the rate of hydrolysis of the least stable substrate, i.e., the activity to hydrolyze NodRlv- $\mathrm{V}\left(\mathrm{C}_{18: 4}, \mathrm{Ac}\right)$ was defined as $100 \%$. The class I chitinase released lipo-tetrasaccharides and lipo-trisaccharides from $\operatorname{NodRlv}-\mathrm{V}\left(\mathrm{C}_{18: 4}, \mathrm{Ac}\right)$ at the ratio $87: 13$. A time course experiment indicated that this ratio remained constant. The fucosylated derivative NodRlv-V( $\mathrm{C}_{18: 4}$, Ac, Fuc) was cleaved to the lipo-trisaccharide at a fourfold lower rate, compared with the hydrolytic rate of non-fucosylated NodRlv- $\mathrm{V}\left(\mathrm{C}_{18: 4}, \mathrm{Ac}\right)$. A protecting effect by the fucosyl group was also found when class I chitinases from other plant species were tested for their substrate specificity toward pentameric NodRlv factors. Moreover, similar results were obtained for a class II chitinase, which belongs, like class I chitinase, to glycosyl hydrolase family 19 (data not shown). These results are reminiscent of the data obtained earlier for the degradation of the structurally different NFs of S. meliloti (Staehelin et al. 1994b; Schultze et al. 1998). It was demonstrated that the sulfate group at the reducing end of NFs increases their stability against hydrolysis by chitinases of family 19 and influences the accessibility of cleavage sites. These chitinases were able to cleave the terminal reducing end monomer from desulfated $\operatorname{NodSm-IV}\left(\mathrm{C}_{16: 2}\right)$ and NodSm$\mathrm{V}\left(\mathrm{C}_{16: 2}\right)$ factors, but not from the sulfated $\operatorname{NodSm-IV}\left(\mathrm{C}_{16: 2}, \mathrm{~S}\right)$ and NodSm-V $\left(\mathrm{C}_{16: 2}, \mathrm{~S}\right)$ molecules.

The differences in stability between fucosylated and nonfucosylated NodRlv factors were studied further by incubating the class I chitinase with an equimolar mixture of NodRlv$\mathrm{V}\left(\mathrm{C}_{18: 4}, \mathrm{Ac}\right)$ and NodRlv-V(C $\mathrm{C}_{18: 4}$, Ac, Fuc) (Fig. 6A and D ). After 15 min of incubation, non-fucosylated NodRlv-V( $\mathrm{C}_{18: 4}$, Ac) was entirely hydrolyzed to lipo-tetramers and lipotrimers, whereas the peak size of the fucosylated NodRlv$\mathrm{V}\left(\mathrm{C}_{18: 4}\right.$, Ac, Fuc) was barely affected (Fig. 6B). Prolonged incubation resulted in a very slow decrease of this peak and even after an overnight incubation degradation of NodRlv$\mathrm{V}\left(\mathrm{C}_{18: 4}\right.$, Ac, Fuc) to the lipo-trimer was incomplete (Fig. 6C). Hence, the effect of the fucosyl group on NF stability was much more pronounced in this experiment with two substrates, compared with the fourfold difference of the hydrolytic rate obtained for fucosylated and non-fucosylated substrates alone (Fig. 5). Since NodRlv-V(C $\left.\mathrm{C}_{18: 4}, \mathrm{Ac}\right)$ was rapidly hydrolyzed by the class I enzyme (Fig. 6B), we suggest that the presence of the non-degradable $\operatorname{NodRlv-IV}\left(\mathrm{C}_{18: 4}, \mathrm{Ac}\right)$ inhibits hydrolysis of NodRlv-V(C $\mathrm{C}_{18: 4}$, Ac, Fuc).
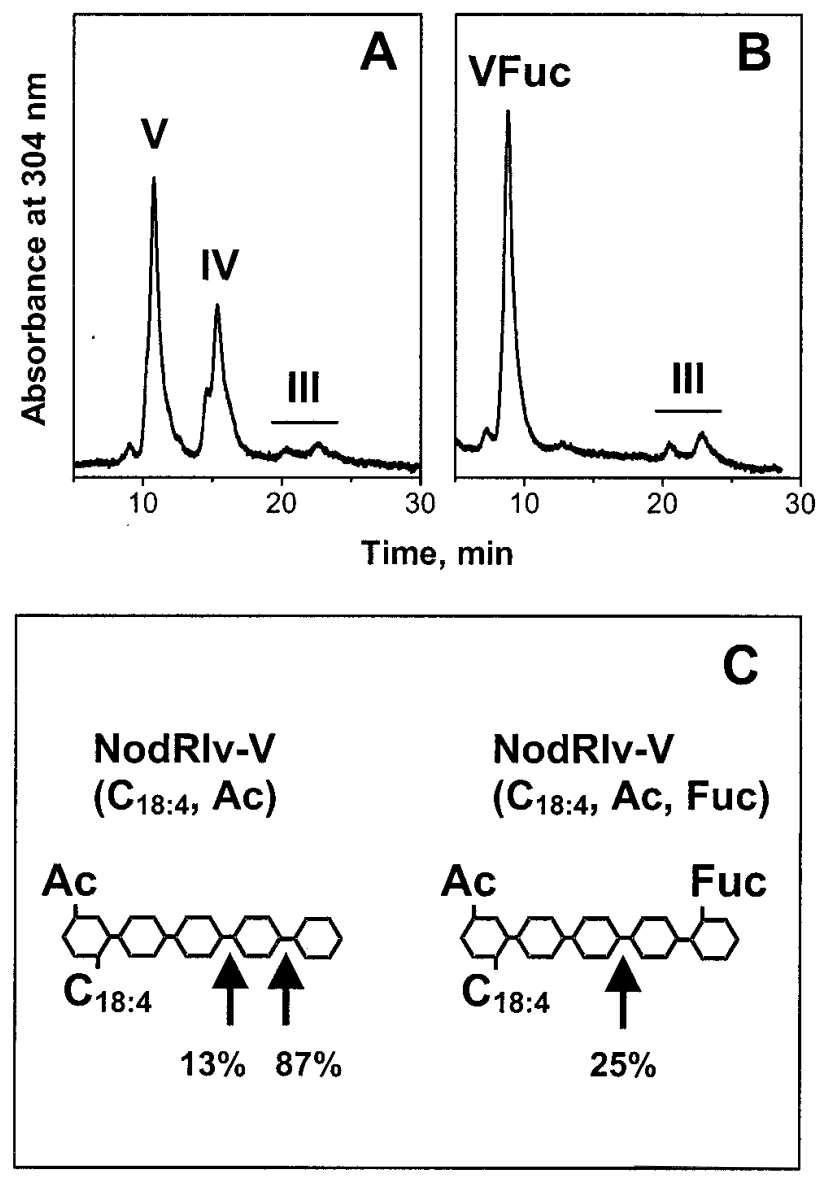

Fig. 5. Hydrolysis of NodRlv factors by tobacco class I chitinase. Highperformance liquid chromatography (HPLC) profiles of pentameric Nod factors (NFs) and their acylated cleavage products after incubation with $0.1 \mu \mathrm{g} \mathrm{ml}^{-1}$ class I chitinase (Chi32) for $1 \mathrm{~h}(\mathbf{A}$ and $\mathbf{B})$. V: NodRlv$\mathrm{V}\left(\mathrm{C}_{18: 4}\right.$, Ac); VFuc: NodRlv-V(C $18: 4$, Ac, Fuc $)$; IV: NodRlv-IV(C $\mathrm{C}_{18: 4}$, Ac); III: NodRlv-III $\left(\mathrm{C}_{18: 4}, \mathrm{Ac}\right)$. A, NodRlv-V(C $\left.\mathrm{C}_{18: 4}, \mathrm{Ac}\right)$ as substrate; NodRlv-IV $\left(\mathrm{C}_{18: 4}, \mathrm{Ac}\right)$ and $\operatorname{NodRlv-III}\left(\mathrm{C}_{18: 4}, \mathrm{Ac}\right)$ as cleavage products. B, NodRlv-V( $\mathrm{C}_{18: 4}$, Ac, Fuc) as substrate; NodRlv-III $\left(\mathrm{C}_{18: 4}\right.$, Ac) as cleavage product. C, Cleavage site preference of class I chitinase. Cleavage sites accessible for the enzyme are indicated by arrows in the schematic structure of the NodRlv factors and their fucosylated derivatives. Values below arrows indicate relative rates of hydrolysis. Ac and Fuc indicate acetylation and fucosylation, respectively. 
A class III chitinase (lys28a from Nicotiana tabacum) showed a different substrate specificity toward NodRlv factors. Incubation of tetrameric $\operatorname{NodRlv-IV}\left(\mathrm{C}_{18: 4}, A c\right)$ with this enzyme resulted in the rapid formation of the lipo-trisaccharide NodRlv-III $\left(\mathrm{C}_{18: 4}\right.$, Ac) (Fig. 7A and C). Lipo-disaccharides were not released, since the $O$-acetyl group at the nonreducing end protected the second glycosidic bond from hydrolysis by class III chitinases, as shown previously for sulfated NodSm factors (Schultze et al. 1998). The fucosylated derivative NodRlv-IV( $\left.\mathrm{C}_{18: 4}, \mathrm{Ac}, \mathrm{Fuc}\right)$ incubated with the class III chitinase remained entirely resistant against hydrolysis, even after prolonged incubation time (up to $24 \mathrm{~h}$ ) (Fig. 7B). Thus, the fucosyl group at the reducing end prevented the class III chitinase from cleaving off a terminal sugar residue at the reducing end, providing complete stability of the tetrameric molecules.

Similarly to the class I chitinase, the class III enzyme hydrolyzed the pentameric NodRlv- $\mathrm{V}\left(\mathrm{C}_{18: 4}, \mathrm{Ac}\right)$ to the tetrasaccharidic NodRlv-IV( $\left.\mathrm{C}_{18: 4}, \mathrm{Ac}\right)$ and to the lipo-trisaccharide NodRlv-III $\left(\mathrm{C}_{18: 4}, \mathrm{Ac}\right)$, while only the lipo-trisaccharide was released from the fucosylated derivative NodRlv-V $\left(\mathrm{C}_{18: 4}, \mathrm{Ac}\right.$, Fuc) (Fig. 7C, chromatograms not shown). Hence, the presence of a fucosyl group at the reducing end also stabilized the adjacent glycosidic bond in pentameric NFs. As shown in Figure $7 \mathrm{C}$, the activity of the class III chitinase to release the lipo-trisaccharide from $\operatorname{NodRlv-IV}\left(\mathrm{C}_{18: 4}, \mathrm{Ac}\right)$ was defined as
$100 \%$. A slightly reduced hydrolytic rate was found for the lipo-trisaccharide formation from the fucosylated pentamer NodRlv- $\mathrm{V}\left(\mathrm{C}_{18: 4}\right.$, Ac, Fuc $)$. Hydrolysis of non-fucosylated NodRlv- $\mathrm{V}\left(\mathrm{C}_{18: 4}\right.$, Ac) resulted in the formation of the lipotetrasaccharide $(25 \%)$ and the lipo-trisaccharide $(36 \%)$. Since the class III chitinase was able to degrade the lipo-tetrasaccharide further, the data for hydrolysis of NodRlv- $\mathrm{V}\left(\mathrm{C}_{18: 4}\right.$, Ac) shown in Figure $7 \mathrm{C}$ reflect a given time point and varied depending on the time of incubation.

These results taken together show that the fucosylation of NodRlv factors conferred protection against hydrolysis by certain plant chitinases in vitro. Chitinases can thus be considered proteins that have the capacity to discriminate between non-modified NFs and their derivatives carrying a reducing end substitution. Similar properties have been postulated for the protein encoded by $s y m 2^{A}$. Further work will be required to investigate the presence and function of chitinases and related proteins in the roots of pea plants carrying or lacking the $\operatorname{sym} 2^{A}$ allele.

\section{MATERIALS AND METHODS}

\section{Plant material and bacterial strains.}

Pea seeds of two near-isogenic lines Rondo-sym $2^{C}$ (the cultivar Rondo) and the backcross line Rondo-sym2 $2^{A}$ (line A
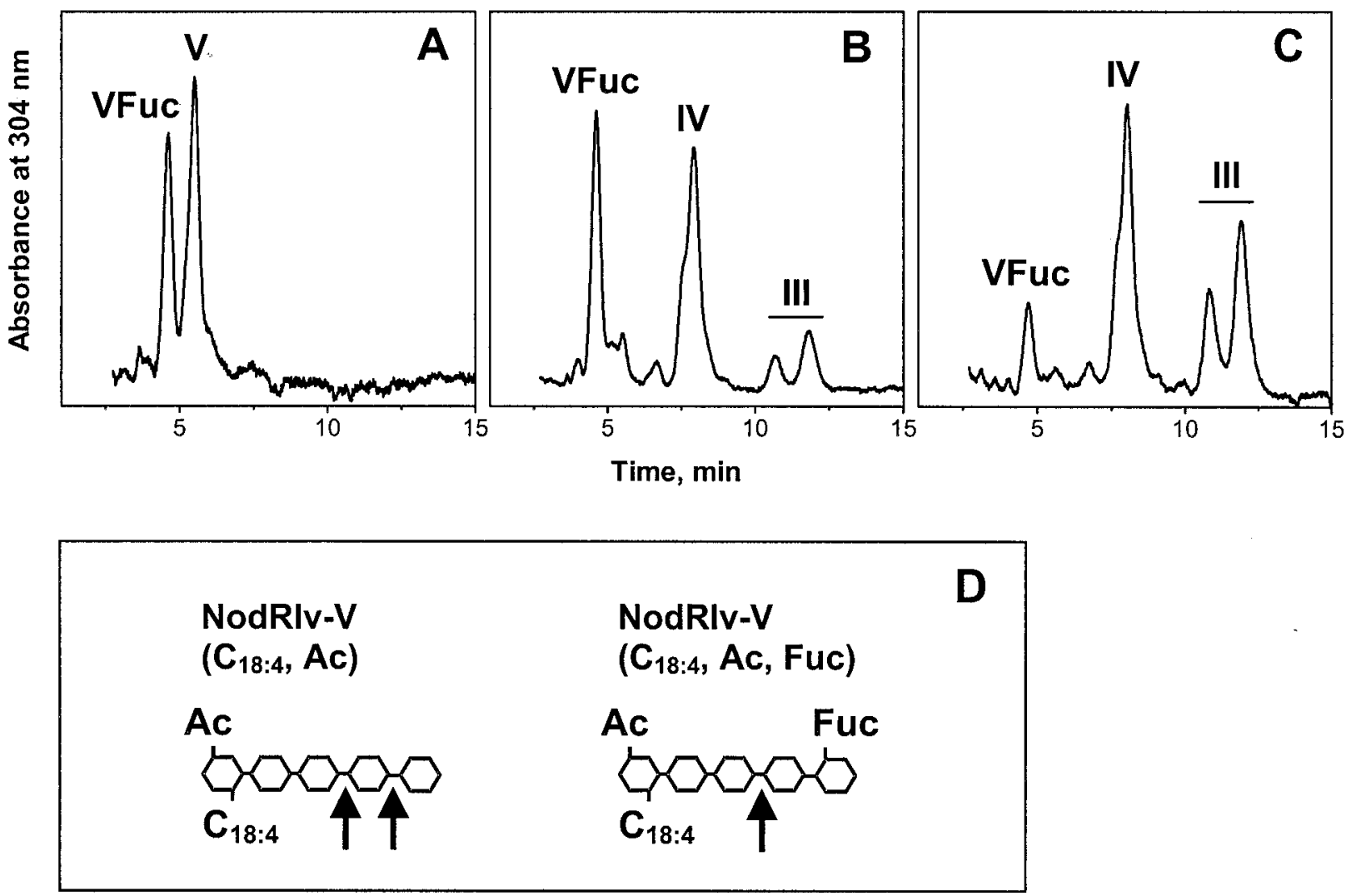

Fig. 6. Substrate preference of tobacco class I chitinase toward an equimolar mixture of NodRlv-V(C $\left.\mathrm{C}_{18: 4}, \mathrm{Ac}\right)$ and $\operatorname{NodRlv-V}\left(\mathrm{C}_{18: 4}, \mathrm{Ac}, \mathrm{Fuc}\right)$. A, B, and C, High-performance liquid chromatography (HPLC) chromatograms: Incubation of NFs was performed with $0.1 \mu \mathrm{g} \mathrm{ml}{ }^{-1}$ of class I chitinase (Chi32). Aliquots of reaction mixture were taken after different time points and analyzed by HPLC. Substrates, V: NodRlv-V(C $\left.C_{18: 4}, A c\right)$; VFuc: NodRlv-V(C $18: 4$, Ac, Fuc). Cleavage products, IV: NodRlv-IV $\left(\mathrm{C}_{18: 4}, \mathrm{Ac}\right)$; III: NodRlv-III $\left(\mathrm{C}_{18: 4}, \mathrm{Ac}\right)$. Shift in elution time of all peaks is due to an increased acetonitrile concentration used for this HPLC analysis (39\%, vs 36\% in previous experiments). A, Substrates without chitinase. B, Incubation with chitinase for 15 min. C, Incubation with chitinase for $16 \mathrm{~h}$. D, Schematic structure of the two NodRlv factors used as substrates. Arrows indicate cleavage sites accessible for class I chitinase. Ac and Fuc indicate acetylation and fucosylation, respectively. 
5.6.9.) were kindly provided by O. A. Kulikova (Wageningen Agricultural University, Wageningen, The Netherlands) and V. E. Tsyganov (All-Russia Research Institute for Agricultural Microbiology, St. Petersburg, Russia). Introgression line A 5.6.9. resulted from crossing of pea line L2150 (cv. Afghanistan) with the European cultivar Rondo-sym $2^{C}$ and three sub-
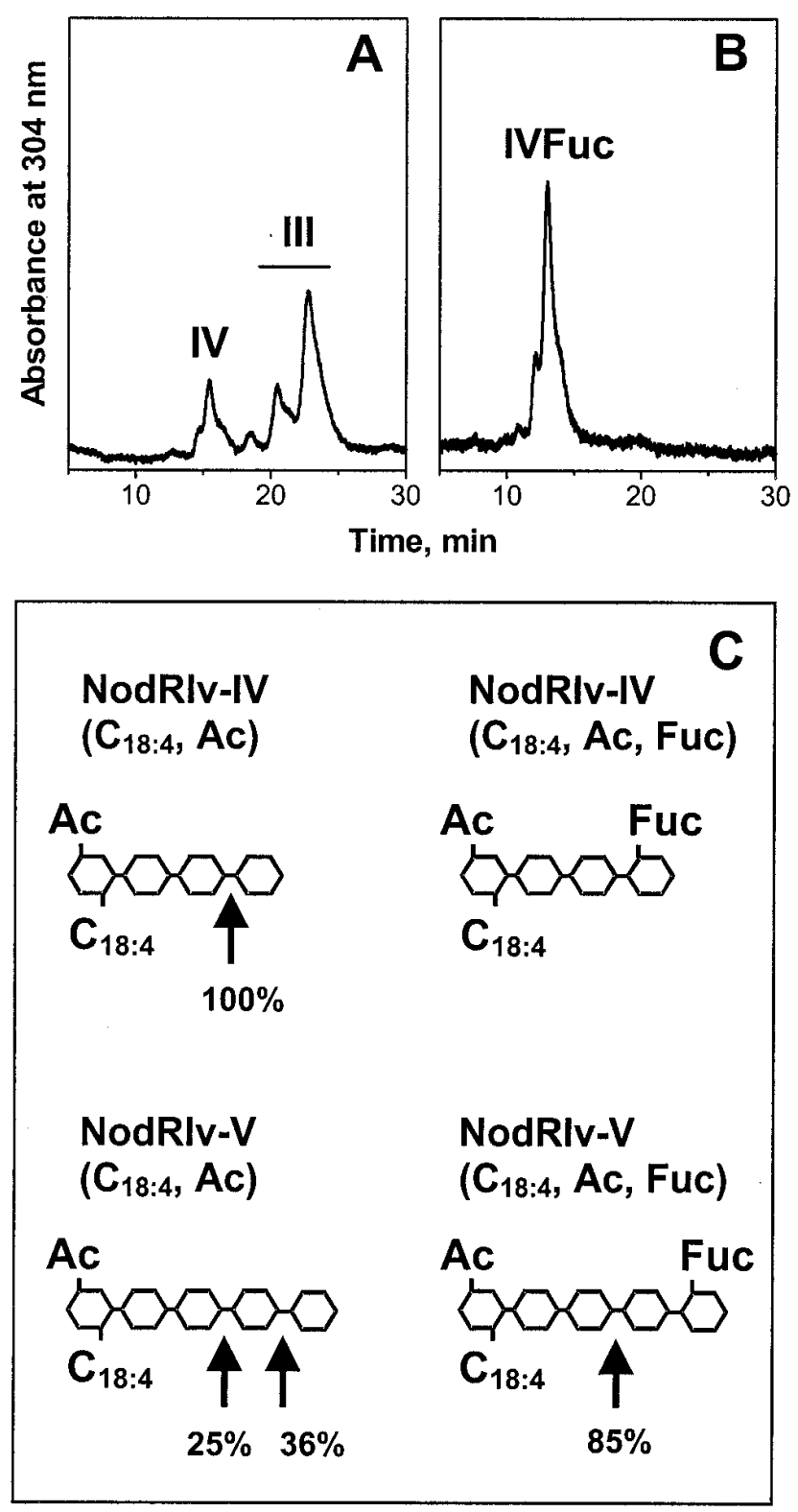

Fig. 7. Hydrolysis of NodRlv factors by tobacco class III chitinase. High-performance liquid chromatography (HPLC) profiles of tetrameric Nod factors (NFs) and their acylated cleavage products after incubation with $0.033 \mu \mathrm{g} \mathrm{ml}^{-1}$ of class III chitinase (lys28a) (A and B). IV: NodRlvIV $\left(\mathrm{C}_{18: 4}, \mathrm{Ac}\right)$; IVFuc: NodRlv-IV(C $\left.\mathrm{C}_{18: 4}, \mathrm{Ac}, \mathrm{Fuc}\right)$; III: NodRlv-III(C $\mathrm{C}_{18: 4}$, Ac). A, Incubation with chitinase for $5 \mathrm{~h}$; NodRlv-IV $\left(\mathrm{C}_{18: 4}, \mathrm{Ac}\right)$ as substrate; NodRlv-III $\left(\mathrm{C}_{18: 4}, \mathrm{Ac}\right)$ as cleavage product. B, Incubation with chitinase for $24 \mathrm{~h}$; NodRlv-IV $\left(\mathrm{C}_{18: 4}, \mathrm{Ac}, \mathrm{Fuc}\right)$ is not hydrolyzed. C, Cleavage site preference of class III chitinase. Cleavage sites accessible for the enzyme are indicated by arrows in the schematic structure of the NodRlv factors and their fucosylated derivatives. Values below arrows indicate relative rates of hydrolysis obtained from incubation with chitinase for 1 h. Ac and Fuc indicate acetylation and fucosylation, respectively. sequent backcrosses to Rondo-sym $2^{C}$ (Kozik et al. 1995). Line A 5.6.9. contains an introgressed region around the $s y m 2^{A}$ gene from cv. Afghanistan at the genetic background of cv. Rondo. Pea seeds were surface sterilized with concentrated sulfuric acid for 5 to $10 \mathrm{~min}$, washed several times with sterile water, and placed for germination on $0.8 \%$ water agar plates at $18^{\circ} \mathrm{C}$ in the dark.

The Rhizobium leguminosarum bv. viciae strain TOM (Winarno and Lie 1979) and the European strain CIAM 1026 (Chetkova and Tikhonovich 1986) were tested for their capacity to enhance the activity of the pea NF hydrolase.

\section{Purification and fucosylation}

of $R$. leguminosarum bv. viciae Nod factors.

NodRlv factors (Spaink et al. 1991) were purified from the overproducing $R$. leguminosarum bv. viciae strain RBL 5799 grown at $28^{\circ} \mathrm{C}$ on a shaker in 3 liters of $\mathrm{B}^{-}$medium (van Brussel et al. 1977) in the presence of $3 \mu \mathrm{M}$ naringenin. NFs were extracted with $n$-butanol, concentrated under reduced pressure, and further pre-purified with a Silica gel 60 column (Machery-Nagel, Düren, Germany). NFs were fractionated on a reverse-phase HPLC column (Waters, Milford, MA; Nova Pak $\mathrm{C}_{18}, 3.9 \times 150 \mathrm{~mm}$, particle size $\left.4 \mu \mathrm{m}\right)$ under isocratic conditions, with $36 \%$ (vol/vol) acetonitrile/water as the mobile phase. The pentameric NodRlv-V $\left(\mathrm{C}_{18: 4}, \mathrm{Ac}\right)$ and the more slowly migrating tetrameric $\operatorname{NodRlv}-\operatorname{IV}\left(\mathrm{C}_{18: 4}, \mathrm{Ac}\right)$ were detected by their absorption at $304 \mathrm{~nm}$.

Fucosylated NF derivatives were obtained by in vitro fucosylation of NodRlv factors with fucosyltransferase (NodZ protein) from Bradyrhizobium japonicum and GDP- $\beta$-Lfucose (Sigma, St. Louis, MO) as a substrate (Quinto et al. 1997). A typical reaction mixture contained NodZ protein (1:50 dilution of the crude extract), 100 to $200 \mu \mathrm{g}$ of purified NodRlv$\operatorname{IV}\left(\mathrm{C}_{18: 4}, \mathrm{Ac}\right)$ or NodRlv-V(C $\left.\mathrm{C}_{18: 4}, \mathrm{Ac}\right), 10 \mathrm{mM} \mathrm{MgCl}_{2}, 2 \mathrm{mM}$ ATP, $20 \mathrm{mM}$ sodium phosphate buffer $(\mathrm{pH} 7.0)$ and $0.45 \mathrm{mM}$ GDP- $\beta$-L-fucose. After incubation at $28^{\circ} \mathrm{C}$ for $24 \mathrm{~h}$, the NF substrate and the fucosylated derivative were fractionated on reverse-phase HPLC with $36 \%$ acetonitrile/water (vol/vol) as the mobile phase. Fractions containing fucosylated derivatives were collected, dried under reduced pressure, and re-dissolved in $100 \%$ DMSO (dimethyl sulfoxide) for further use.

For a control experiment, the in vitro reaction was performed with GDP- $\beta-\mathrm{L}-\left[1-{ }^{14} \mathrm{C}\right]$ fucose $(292 \mathrm{mCi} / \mathrm{mmol}$; Amersham Pharmacia Biotech, Uppsala, Sweden) and $10 \mu \mathrm{g}$ of NodRlv-IV $\left(\mathrm{C}_{18: 4}, \mathrm{Ac}\right)$ or $\operatorname{NodRlv-V}\left(\mathrm{C}_{18: 4}, \mathrm{Ac}\right)$, respectively. After $n$-butanol extraction and concentration, NFs were chromatographed on reverse-phase $\mathrm{C}_{18}$-coated TLC plates (Sigma) in $50 \%(\mathrm{vol} / \mathrm{vol})$ acetonitrile/water. Plates were developed with the PhosphorImager system and Image Quant software (Molecular Dynamics, Sunnyville, CA).

\section{Pretreatment of pea seedlings with rhizobia and NFs.}

Three or four days after sterilization, roots of germinated pea seedlings were either pretreated with a rhizobial culture $\left(A_{660}=0.05\right)$ or with Jensen medium (van Brussel et al. 1982) containing purified NFs. The NodRlv factors and their fucosylated derivatives were tested at different concentrations. NodSm-V $\left(\mathrm{C}_{16: 2}, \mathrm{~S}\right)$ from Sinorhizobium meliloti (Schultze et al. 1992) was used at a concentration of $10^{-6} \mathrm{M}$. Control plants were mock treated with Jensen medium without NFs. Plants were incubated at $18^{\circ} \mathrm{C}$ for $20 \mathrm{~h}$ in the dark. 


\section{Measurement of the pea NF hydrolase activity.}

The assay originally developed for $M$. sativa plants (Staehelin et al. 1995) was modified for pea seedlings. After pretreatment, the pea plants were individually transferred into 1-ml plastic syringes filled with $400 \mu \mathrm{l}$ of Jensen medium supplemented with $5 \mu \mathrm{M}$ NodSm-V(C $\left.\mathrm{C}_{16: 2}, \mathrm{~S}\right)$. After $4 \mathrm{~h}$ of incubation at $24^{\circ} \mathrm{C}$, the NodSm factor and acylated cleavage products in the culture medium were extracted with $n$ butanol, concentrated, and analyzed by reverse-phase HPLC at $220 \mathrm{~nm}$ with $35 \%$ acetonitrile/water containing $40 \mathrm{mM}$ ammonium acetate as the mobile phase. The pea roots released two cleavage products from $\operatorname{NodSm}-\mathrm{V}\left(\mathrm{C}_{16: 2}, \mathrm{~S}\right)$, the lipo-trisaccharide $\operatorname{NodSm-III(}\left(\mathrm{C}_{16: 2}\right)$ and the lipo-disaccharide NodSm-II $\left(\mathrm{C}_{16: 2}\right)$. To compare the data obtained in different experimental series, the NF-inducible formation of lipodisaccharide was corrected with the non-inducible lipo-trisaccharide formation as an internal standard.

\section{Hydrolysis of NodRlv factors by purified chitinases.}

For the in vitro experiments, plant chitinases were incubated with $2 \mathrm{nmol}$ of purified tetrameric $\operatorname{NodRlv}-\operatorname{IV}\left(\mathrm{C}_{18: 4}\right.$, Ac), fucosylated NodRlv-IV $\left(\mathrm{C}_{18: 4}\right.$, Ac, Fuc), pentameric

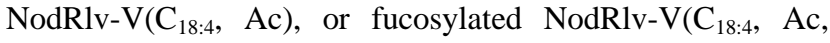
Fuc). The purified chitinases (lys28a from Nicotiana tabacum; Brunner et al. 1998; Chi32 from Nicotiana tabacum; Legrand et al. 1987) were stored on ice and a decrease of specific activity upon storage was observed; therefore, values for specific activities need to be interpreted with caution and cannot be directly compared with those of Schultze et al. (1998). The reaction was performed in $0.2 \mathrm{M}$ sodium acetate buffer at $\mathrm{pH}$ 4.5 for the class III chitinase (lys28a) and at $\mathrm{pH} 5.0$ for the class I chitinase (Chi32). The enzyme concentration in the reaction mixture was $0.033 \mu \mathrm{g} \mathrm{ml}^{-1}$ for the class III chitinase and $0.1 \mu \mathrm{g} \mathrm{ml}^{-1}$ for the class I chitinase. The reaction mixture was incubated at $37^{\circ} \mathrm{C}$ for different periods. Samples were extracted twice with half-volume of $n$-butanol, then the butanol phases were combined, dried in a speed-vac evaporator, and finally re-dissolved in $1 \mu \mathrm{l}$ of DMSO. NodRlv substrates and the acylated cleavage products were fractionated on a $\mathrm{C}_{18}$ reversephase HPLC column at $304 \mathrm{~nm}$ under isocratic conditions with 36 or $39 \%$ acetonitrile/water as a mobile phase. Hydrolytic rates of NFs were determined by the integration of peak areas of the acylated cleavage products. To compare the rate of hydrolysis for the different NFs, the relative rate of hydrolysis for each glycosidic bond was determined. For each enzyme, the rate of hydrolysis of the least stable NF was defined as $100 \%$.

\section{ACKNOWLEDGMENTS}

This work was supported in part by INTAS (project no. 96-1371), RFBR (project no. 96-04-50361 to A. O. O.) and the Netherlands Organisation for Scientific Research (NWO). We thank F. Brunner and M. Legrand (Institut de Biologie Moléculaire des Plantes, CNRS, Strasbourg, France) for purified chitinases, O. A. Kulikova and V. E. Tsyganov (RIAM) for the pea seeds, and M. Harteveld (Leiden University) for the preparation of the NodZ protein. We are grateful to B. J. J. Lugtenberg for continuous help and support for presentation of this work at the 3rd European Nitrogen Fixation Conference (Lunteren, The Netherlands).

\section{LITERATURE CITED}

Brunner, F., Stintzi, A., Fritig, B., and Legrand, M. 1988. Substrate specificities of tobacco chitinases. Plant J. 14:225-234.
Chetkova, S. A., and Tikhonovich, I. A. 1986. Isolation and analysis of Rhizobium leguminosarum strains which are effective on Afghanistan peas. (In Russian). Microbiologia 55:143-147.

Collinge, D. B., Kragh, K. M., Mikkelsen, J. D., Nielsen, K. K., Rasmussen, U., and Vad, K. 1993. Plant chitinases. Plant J. 3:31-40.

De Jong, A. J., Cordewener, J., Lo Schiavo, F., Terzi, M., Vandekerckhove, J., van Kammen, A., and De Vries, S. C. 1992. A carrot somatic embryo mutant is rescued by chitinase. Plant Cell 4:425-433.

Dénarié, J., Debellé, F., and Promé, J.-C. 1996. Rhizobium lipo-chitooligosaccharide nodulation factors: Signaling molecules mediating recognition and morphogenesis. Annu. Rev. Biochem. 65:503-535.

Firmin, J. L., Wilson, K. E., Carlson, R. W., Davies, A. E., and Downie, J. A. 1993. Resistance to nodulation of cv. Afghanistan peas is overcome by nodX, which mediates an O-acetylation of the Rhizobium leguminosarum lipo-oligosaccharide nodulation factor. Mol. Microbiol. 10:351-360.

Geurts, R., Heidstra, R., Hadri, A. E., Downie, J. A., Franssen, H., van Kammen, A., and Bisseling, T. 1997. Sym2 of pea is involved in a nodulation factor-perception mechanism that controls the infection process in the epidermis. Plant Physiol. 115:351-359.

Goormachtig, S., Lievens, S., Van de Velde, W., Van Montagu, M., and Holsters, M. 1998. Srchi13, a novel early nodulin from Sesbania rostrata, is related to acidic class III chitinases. Plant Cell 10:905-915.

Heidstra, R., Geurts, R., Franssen, H., Spaink, H. P., van Kammen, A., and Bisseling, T. 1994. Root hair deformation activity of nodulation factors and their fate on Vicia sativa. Plant Physiol. 105:787-797.

Henrissat, B. 1991. A classification of glycosyl hydrolases based on amino acid sequence similarities. Biochem. J. 280:309-316.

Kozik, A., Heidstra, R., Horvath, B., Kulikova, O., Tikhonovich, I., Ellis, T. H. N., Van Kammen, A., Lie, T. A., and Bisseling, T. 1995. Pea lines carrying sym 1 or sym 2 can be nodulated by Rhizobium strains containing nodX; sym1 and sym2 are allelic. Plant Sci. 108:41-49.

Krishnan, H. B., Kim, K. Y., and Krishnan, A. H. 1999. Expression of a Serratia marcescens chitinase gene in Sinorhizobium fredii USDA191 and Sinorhizobium meliloti RCR2011 impedes soybean and alfalfa nodulation. Mol. Plant-Microbe Interact. 12:748-751.

Legrand, M., Kauffmann, S., Geoffroy, P., and Fritig, B. 1987. Biological function of pathogenesis-related proteins are chitinases. Proc. Natl. Acad. Sci. USA 84:6750-6754.

Lie, T. A. 1984. Host genes in Pisum sativum L. conferring resistance to European Rhizobium leguminosarum strains. Plant Soil 82:415-425.

Minic, Z., Brown, S., De Kouchkovsky, Y., Schultze, M., and Staehelin, C. 1998. Purification and characterization of a novel chitinaselysozyme, of another chitinase, both hydrolysing Rhizobium meliloti Nod factors, and of a pathogenesis-related protein from Medicago sativa roots. Biochem. J. 332:329-335.

Neuhaus, J.-M., Fritig, B., Linthorst, H. J. M., Meins, F., Mikkelsen, J. D., and Ryals, J. 1996. A revised nomenclature for chitinase genes. Plant Mol. Biol. Rep. 14:102-104.

Ovtsyna, A. O., Geurts, R., Bisseling, T., Lugtenberg, B. J. J., Tikhonovich, I. A., and Spaink, H. P. 1998. Restriction of host range by the sym 2 allele of Afghan pea is non-specific for the type of modification at the reducing terminus of nodulation signals. Mol. PlantMicrobe Interact. 11:418-422.

Ovtsyna, A. O., Rademaker, G.-J., Esser, E., Weinman, J., Rolfe, B. G., Tikhonovich, I. A., Lugtenberg, B. J. J., Thomas-Oates, J. E., and Spaink, H. P. 1999. Comparison of characteristics of the nodX genes from various Rhizobium leguminosarum strains. Mol. Plant-Microbe Interact. 12:252-258.

Parniske, M., Schmidt, P. E., Kosch, K., and Müller, P. 1994. Plant defense responses of host plants with determinate nodules induced by EPS-defective exoB mutants of Bradyrhizobium japonicum. Mol. Plant-Microbe Interact. 7:631-638.

Quinto, C., Wijfjes, A. H. M., Bloemberg, G. V., Blok-Tip, L., LopezLara, I. M., Lugtenberg, B. J. J., Thomas-Oates, J. E., and Spaink, H. P. 1997. Bacterial nodulation protein NodZ is a chitin oligosaccharide fucosyltransferase which can also recognize related substrates of animal origin. Proc. Natl. Acad. Sci. USA 94:4336-4341.

Schultze, M., and Kondorosi, A. 1998. Regulation of symbiotic root nodule development. Annu. Rev. Genet. 32:33-57.

Schultze, M., Quiclet-Sire, B., Kondorosi, E., Virelizier, H., Glushka, J. N., Endre, G., Géro, S. D., and Kondorosi, A. 1992. Rhizobium meliloti produces a family of sulfated lipooligosaccharides exhibiting different degrees of plant host specificity. Proc. Natl. Acad. Sci. USA 89:192-196. 
Schultze, M., Staehelin, C., Brunner, F., Genetet, I., Legrand, M., Fritig, B., Kondorosi, E., and Kondorosi, A. 1998. Plant chitinase/lysozyme isoforms show distinct substrate specificity and cleavage site preference towards lipochitooligosaccharide Nod signals. Plant J. 16:571580.

Spaink, H. P. 1996. Regulation of plant morphogenesis by lipo-chitin oligosaccharides. Crit. Rev. Plant Sci. 15:559-582.

Spaink, H. P. , Sheeley, D. M., Van Brussel, A. A. N., Glushka, J., York, W. S., Tak, T., Geiger, O., Kennedy, E. P. Reinhold, V. N., and Lugtenberg, B. J. J. 1991. A novel highly unsaturated fatty acid moiety of lipo-oligosaccharide signals determines host specificity of $R h i$ zobium. Nature 354:125-130.

Staehelin, C., Granado, J., Müller, J., Wiemken, A., Mellor, R. B., Felix, G., Regenass, M., Broughton, W. J., and Boller, T. 1994a. Perception of Rhizobium nodulation factors by tomato cells and inactivation by root chitinases. Proc. Natl. Acad. Sci. USA 91:2196-2200.

Staehelin, C., Müller, J., Mellor, R. B., Wiemken, A., and Boller, T. 1992. Chitinase and peroxidase in effective (fix ${ }^{+}$) and ineffective $\left(\right.$ fix $\left.^{-}\right)$soybean nodules. Planta 187:295-300.

Staehelin, C., Schultze, M., Kondorosi, E., and Kondorosi, A. 1995. Lipo-chitooligosaccharide nodulation signals from Rhizobium meliloti induce their rapid degradation by the host plant alfalfa. Plant Physiol. 108:1607-1614.

Staehelin, C., Schultze, M., Kondorosi, E., Mellor, R. B., Boller, T., and
Kondorosi, A. 1994b. Structural modifications in Rhizobium meliloti Nod factors influence their stability against hydrolysis by root chitinases. Plant J. 5:319-330.

Staehelin, C., Vanney, M., Foucher, F., Kondorosi, E., Schultze, M., and Kondorosi, A. 1997. Degradation of nodulation signals from Rhizobium meliloti by its host plants. Pages 43-46 in: Biological Fixation of Nitrogen for Ecology and Sustainable Agriculture. A. Legocki, H. Bothe, and A. Pühler, eds. Springer, Berlin.

van Brussel, A. A. N., Planque, K., and Quispel, A. 1977. The wall of Rhizobium leguminosarum in bacteroid and free-living forms. J. Gen. Microbiol. 101:51-56.

van Brussel, A. A. N., Tak, T., Wetselaar, A., Pees, E., and Wijffelman, C. A. 1982. Small Leguminosae as test plants for nodulation of Rhizobium leguminosarum and other rhizobia and agrobacteria harbouring a leguminosarum Sym plasmid. Plant Sci. Lett. 27:317-325.

Vasse, J., de Billy, F., and Truchet, G. 1993. Abortion of infection during the Rhizobium meliloti-alfalfa symbiotic interaction is accompanied by a hypersensitive reaction. Plant J. 4:555-566.

Winarno, R., and Lie, T. A. 1979. Competition between Rhizobium strains in nodule formation: Interaction between nodulating and nonnodulating strains. Plant Soil 51:135-142.

Xie, Z.-P., Staehelin, C., Wiemken, A., Broughton, W. J., Müller, J., and Boller, T. 1999. Symbiosis-stimulated chitinase isoenzymes of soybean (Glycine max (L.) Merr.). J. Exp. Bot. 50:327-333. 